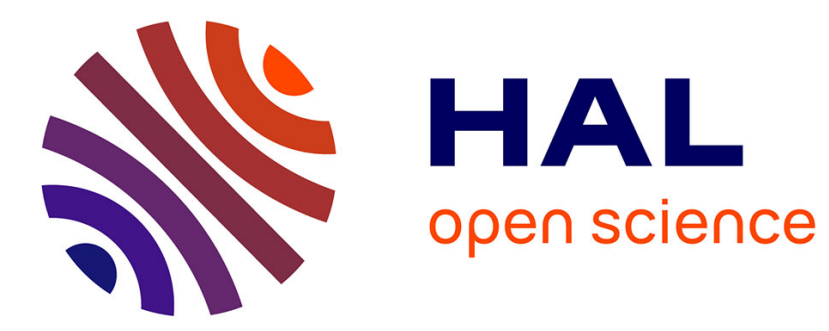

\title{
Can one bind three electrons with a single proton?
}

Dario Bressanini, Raymond Brummelhuis, Pierre Duclos, Renaud Ruamps

\section{To cite this version:}

Dario Bressanini, Raymond Brummelhuis, Pierre Duclos, Renaud Ruamps. Can one bind three electrons with a single proton?. Workshop on Critical Stability of Few-Body Quantum Systems, Oct 2008, Erice, Italy. pp.173-177, 10.1007/s00601-009-0018-7 . hal-00364364

\section{HAL Id: hal-00364364 https://hal.science/hal-00364364}

Submitted on 25 Feb 2009

HAL is a multi-disciplinary open access archive for the deposit and dissemination of scientific research documents, whether they are published or not. The documents may come from teaching and research institutions in France or abroad, or from public or private research centers.
L'archive ouverte pluridisciplinaire HAL, est destinée au dépôt et à la diffusion de documents scientifiques de niveau recherche, publiés ou non, émanant des établissements d'enseignement et de recherche français ou étrangers, des laboratoires publics ou privés. 
Few-Body Systems 0, 1-6 (2009)

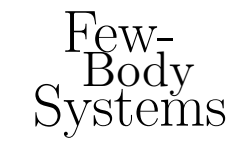

(C) by Springer-Verlag 2009 Printed in Austria

\section{Can one bind three electrons with a single proton?*}

D. Bressanini ${ }^{1 * *}$, R. Brummelhuis ${ }^{2 * * *,}$, P. Duclos ${ }^{3}{ }^{\dagger \dagger}$, R. Ruamps ${ }^{4}$ B $^{* \dagger}$

${ }^{1}$ Dipartimento di Scienze Chimiche, Fisiche e Matematiche - Universita'dell'Insubria, Italy

${ }^{2}$ Université de Reims, FRE 3111, Département de mathématique et informatique, Moulin de la Housse, BP 103951687 Reims cedex 2, France

${ }^{3}$ Centre de Physique Théorique de Marseille UMR 6207 - Unité Mixte de Recherche du CNRS et des Universités Aix-Marseille I, Aix-Marseille II et de l' Université du Sud Toulon-Var Laboratoire affilié à la FRUMAM, Luminy Case 907, 13288 Marseille cedex 9, France

47 place des Pradettes 31100 Toulouse

Abstract. Of course not for an ideal $\mathrm{H}^{--}$atom. But with the help of an intense homogeneous magnetic field $B$, the question deserves to be reconsidered. It is known (see e.g. [BSY, BD]) that as $B \rightarrow \infty$ and in the clamped nucleus approximation, this ion is described by a one dimensional Hamiltonian

$$
\sum_{i=1}^{N}-\frac{\Delta_{i}}{2}-Z \delta\left(x_{i}\right)+\sum_{1 \leq i<j \leq N} \delta\left(x_{i}-x_{j}\right) \quad \text { acting in } L^{2}\left(\mathbb{R}^{3}\right)
$$

where $N=3, Z=1$ is the charge of the nucleus, and $\delta$ stands for the well known "delta" point interaction. We present an extension of the "skeleton method", see [CDR1, CDR2], to the case of three degree of freedom . This is a tool, that we learn from $[\mathrm{R}]$ for the case $N=2$, which reduces the spectral analysis of (1) to determining the kernel a system of linear integral operators acting on the supports of the delta interactions. As an application of this method we present numerical results which indicates that (1) has a bound state for $Z=1$ and $N=3$.

\footnotetext{
*Article based on the presentation by P. Duclos at the Fifth Workshop on Critical Stability, Erice, Sicily, Received December 16, 2008; Accepted January 27, 2009.

**E-mail address: dario.bressanini@uninsubria.it

${ }^{* * *}$ Alternative address: Birkbeck, University of London, School of Economics, Mathematics and Statistics, Malet Street, WC1E 7HX, London, UK

${ }^{\dagger}$ E-mail address: raymond.brummelhuis@univ-reims.fr

${ }^{\dagger \dagger}$ E-mail address: duclos@univ-tln.fr

${ }^{\dagger \dagger}$ E-mail address: renaudruamps@free.fr
} 


\section{Introduction}

It is known by Lieb's inequality [L] that an atom with a nucleus charge $Z$ and an infinite nuclear mass can bind at most $N$ electrons with $N<2 Z+1$, so that the answer to the question posed in the title is no for such an atom. Even it is strongly believed and numerically and experimentally verified that the bound should be $N \leq Z+1$. However if one puts the atom in an intense homogeneous magnetic field the number of electrons that can be bound by a nuclear charge $Z$ may increase drastically. The Hamiltonian in such conditions reads

$$
H^{B}(N, Z):=\sum_{j=1}^{N} \frac{\left(-i \nabla_{j}-\frac{1}{2} \mathbb{B} \wedge r_{j}\right)^{2}}{2 m}-\frac{Z}{\left|r_{j}\right|}+\sum_{1 \leq j<k \leq N} \frac{1}{\left|r_{j}-r_{k}\right|}
$$

where $r_{j}$ is the position of the $j^{\text {th }}$ electron with respect to the fixed nucleus and $\mathbb{B}$ is a constant magnetic field of strength $B$. If one introduces the critical number of electrons as ( spect $_{d} X$ stands for discrete spectrum of $X$ )

$$
N_{c}(B, Z):=\max \left\{N, \operatorname{spect}_{d} H^{B}(N, Z) \neq \emptyset\right\}
$$

it was shown in [LSY, Th. 1.5] that

$$
\liminf _{Z \& \frac{B}{Z^{3}} \rightarrow \infty} \frac{N_{c}(B, Z)}{Z} \geq 2
$$

and they conjectured that the above limit should be indeed 2 . The main motivation of the present work is to start the study of the ratio $N / Z_{c}(B, N)$ with

$$
Z_{c}(B, N):=\inf \left\{Z, \operatorname{spect}_{d} H^{B}(N, Z) \neq \emptyset\right\}
$$

for finite $Z$ and $N$ and large $B$ in order to explore how many electrons a charge $Z$ can bind thanks to this strong magnetic field.

The mechanism by which this binding enhancement occurs is well understood: high intensity magnetic fields make the atom one dimensional. It has even been shown, see [BD, Th.1.5], that $H^{B}(N, Z)$, restricted to any fixed total angular momentum along the magnetic field axis, is asymptotic in the norm resolvent sense to a rescaled version of (1) as $B \rightarrow \infty$, at least for spectral parameters in a suitable neighbourhood of the bottom of the spectrum of $H^{B}(N, Z)$. Thus if we prove that (1) has a discrete eigenvalue for a given charge $Z$, we can guarantee that this remains true for $H^{B}(N, Z)$, for a large enough intensity of the magnetic field $\mathbb{B}$. To appreciate the importance of this binding enhancement we shall compare the ratio $N / Z_{c}(B=\infty, N)$ with the same ones for zero magnetic field with bosonic statistics, see Table 1.

As often in these atomic problems it is convenient to work with the following rescaled version of (1)

$$
h(N, \lambda):=\sum_{i=1}^{N}-\frac{\Delta_{i}}{2}-\delta\left(x_{i}\right)+\lambda \sum_{1 \leq i<j \leq N} \delta\left(x_{i}-x_{j}\right), \quad \lambda:=\frac{1}{Z}
$$


We also remark that to prove the existence of a bound state for $N=3$ we need only consider $h:=h(3, Z)$ in the bosonic sector, see [BD, Th. 1.8 and the discussion in $\S \mathrm{IX}]$, providing we take the part of $H^{B}(N, Z)$ with total angular momentum with respect to the magnetic field axis $\mathbb{M} \geq \frac{1}{2} N(N-1)$ with $N=3$, i.e. $\mathbb{M} \geq 3$.

\section{Simple variational approaches}

We define a critical value of $Z$ attached to (1) as follows

$$
\hat{Z}(N):=\inf \left\{Z, \operatorname{spect}_{\mathrm{d}} h\left(N, \lambda=\frac{1}{Z}\right) \neq \emptyset\right\}
$$

which may be considered according to the discussion in $\S 1$ as $Z_{c}(B=\infty, N)$. It is natural to try to find a wave function $\Psi$ so that $(h \Psi, \Psi)$ is below $\Sigma(Z=1 / \lambda)$, the infimum of the essential spectrum of $h ; \Sigma(Z)$, which, by the HVZ theorem, is equal to $\inf h(2, \lambda)$, is known only numerically but thanks to the skeleton methods of Rosenthal, [R, Table I], the curve $Z \rightarrow \Sigma(Z)$ is known with a fairly good accuracy, sufficient for our purposes, see the solid curve in Figure 1 below. The trial function we take is $\Psi(x):=P_{\text {bose }} \prod_{i=1}^{3} a_{i} e^{-\left|a_{i}\right| x_{i}}, a_{i}>0$ where $P_{\text {bose }}$ denotes the projector on the functions which are invariant under the exchange of particles. With $a_{1}=a_{2}=a_{3}=a$ one gets: $(h \Psi, \Psi)=\frac{3}{2} a^{2}-3 a+\frac{3 \lambda}{2} a$ and optimizing over $a$ leads to $(h \Psi, \Psi)=-\frac{3}{8}(\lambda-2)^{2}$. Requiring that this value is below $\Sigma$ gives $\hat{Z}_{c}(3) \leq 1.75$. Then with a two parameter function with $a_{1}=a_{2}=a$ and $a_{3}=b$ we get

$$
(h \Psi, \Psi)=\frac{2 a^{3} b+4 a^{2} b^{2}}{(a+b)^{2}}-\frac{4 a^{2} b}{(a+b)^{2}}-\frac{4 a b}{a+b}+\lambda\left(\frac{8 a^{2} b}{(3 a+b)(a+b)}+\frac{a b}{a+b}\right) .
$$

Looking for the highest possible value of $\lambda$ so that $(h \Psi, \Psi)$ is below $\Sigma$ by a "contour plot", gives $\hat{Z}_{c}(3) \leq 1.45$. We have also done the computation with three parameters and obtained $\hat{Z}_{c}(3) \leq 1.32$. One could of course try more elaborate trial functions; we prefer instead to switch to:

\section{The skeleton method}

Let $\tau_{i}$, resp. $\tau_{i, j}$ denote the trace (restriction) operators to the plane $x_{i}=0$, resp. $x_{i}=x_{j}$. To identify these planes with $\mathbb{R}^{2}$, we choose an oriented basis in each of them as follows: let $\left\{A_{1}, A_{2}, A_{3}\right\}$ denote the canonical basis of $\mathbb{R}^{3}$

\begin{tabular}{c|c|c|c} 
equ. & basis & normal & trace op. \\
\hline$x_{1}=0$ & $b^{(1)}:=\left\{A_{2}, A_{3}\right\}$ & $A_{1}$ & $\tau_{1}$ \\
$x_{2}=0$ & $b^{(2)}:=\left\{A_{3}, A_{1}\right\}$ & $A_{2}$ & $\tau_{2}$ \\
$x_{3}=0$ & $b^{(3)}:=\left\{A_{1}, A_{2}\right\}$ & $A_{3}$ & $\tau_{3}$ \\
$x_{1}=x_{2}$ & $b^{(4)}:=\left\{\frac{A_{1}+A_{2}}{\sqrt{2}}, A_{3}\right\}$ & $\frac{-A_{2}+A_{1}}{\sqrt{2}}=: A_{4}$ & $\tau_{4}:=\tau_{1,2}$ \\
$x_{2}=x_{3}$ & $b^{(5)}:=\left\{\frac{A_{2}+A_{3}}{\sqrt{2}}, A_{1}\right\}$ & $\frac{-A_{3}+A_{2}}{\sqrt{2}}=: A_{5}$ & $\tau_{5}:=\tau_{2,3}$ \\
$x_{3}=x_{1}$ & $b^{(6)}:=\left\{\frac{A_{3}+A_{1}}{\sqrt{2}}, A_{2}\right\}$ & $\frac{-A_{1}+A_{3}}{\sqrt{2}}=: A_{6}$ & $\tau_{6}:=\tau_{3,1}$
\end{tabular}


and define: $\left(\tau_{i} \Psi\right)(s)=\psi\left(s_{1} b_{1}^{(i)}+s_{2} b_{2}^{(i)}\right)$. Let $\mathcal{H}^{1}\left(\mathbb{R}^{3}\right)$ denote the usual Sobolev space and $\tau: \mathcal{H}^{1}\left(\mathbb{R}^{3}\right) \rightarrow \oplus_{i=1}^{6} L^{2}\left(\mathbb{R}^{2}\right)$ be defined by $\tau \Psi:=$ $\left(\tau_{1} \Psi, \tau_{2} \Psi, \tau_{3} \Psi, \tau_{1,2} \Psi, \tau_{2,3} \Psi, \tau_{3,1} \Psi\right)$. Let $h_{0}:=-\Delta / 2$ acting on $L^{2}\left(\mathbb{R}^{3}\right)$ and $r_{0}(E):=$ $\left(h_{0}-E\right)^{-1}$ its resolvent. One can rewrite $h:=h(3, \lambda)$ in the sense of quadratic forms as $h=h_{0}+\tau^{\star} g \tau$ where $g$ stands for the $6 \times 6$ diagonal matrix with diagonal $(-1,-1,-1, \lambda / \sqrt{2}, \lambda / \sqrt{2}, \lambda / \sqrt{2})$. If we let $r(E):=(h-E)^{-1}$ then one has using the second resolvent equation that $r(E)=r_{0}(E)-r_{0}(E) \tau^{\star} S(E)^{-1} \tau r_{0}(E)$ with $S(E):=g^{-1}+K(E)$ and $K(E):=\tau r_{0}(E) \tau^{\star}$. We shall use a theorem (see e.g. [CDR2, Th. 2.3] for a proof) which asserts that

$$
\Sigma(Z)>-k^{2} \in \operatorname{spect}_{\mathrm{d}} h \Longleftrightarrow \operatorname{ker} S\left(-k^{2}\right) \neq\{0\} .
$$

It will be easier to work in the Fourier image and to perform a scaling so that $S\left(-k^{2}\right)$ appears to be unitarily equivalent to $k\left(g^{-1} k+\widehat{K(-1)}\right)$. In view of (4) we have to find $k>\sqrt{-\Sigma}$ so that ker $g^{-1} k+\widehat{K(-1)} \neq\{0\}$ where the hat stands for the Fourier transform. Such a spectral problem in $k$ is sometimes call an operator pencil. We shall call $g^{-1} k+\widehat{K(-1)}$ the skeleton of $h . \widehat{K(-1)}$ is a $6 \times 6$ matrix of integral operators on $L^{2}\left(\mathbb{R}^{2}\right)$. To give a flavour we explicitly write down two of them; with the notations: $T_{0}:=\widehat{K_{i, i}(-1)}, T_{i, j}:=\widehat{\tau_{i} r_{0}(-1)} \tau_{j}^{\star}$

$$
T_{0}(p, q)=\widehat{\tau_{i}} \widehat{r_{0}(-1)} \tau_{i}^{\star}=\frac{\delta(p-q)}{\sqrt{p^{2}+2}}, \quad T_{1,2}(p, q)=\frac{\delta\left(q_{1}-p_{2}\right)}{\pi\left(\left(p_{1}^{2}+p_{2}^{2}+q_{2}^{2}\right)+2\right)} .
$$

It turns out that these integral operators $T_{i, j}$ depend mostly on the angle between the planes on which $\tau_{i}$ and $\tau_{j}$ operate their restriction. That is why we adopt the following notations: $T_{\frac{\pi}{2}}=T_{1,2}, T_{\frac{\pi}{4}}=T_{1,4}, \tilde{T}_{\frac{\pi}{2}}:=T_{1,5}, T_{\frac{\pi}{3}}=T_{4,5}$. Thanks to the fact that we are working in the bosonic sector, the skeleton reduces by symmetry to

$$
\left(\begin{array}{cc}
-k+T_{0}+2 T_{\frac{\pi}{2}}^{\sharp} & 3 T_{\frac{\pi}{4}}^{\sharp} \\
3\left(T_{\frac{\pi}{4}}^{\sharp}\right)^{\star} & \frac{\sqrt{2}}{\lambda} k+T_{0}+2 T_{\frac{\pi}{3}}
\end{array}\right)
$$

with $(\varepsilon \psi(p, q):=\psi(q, p)) T_{\frac{\pi}{2}}^{\sharp}:=\frac{1}{2}\left(T_{\frac{\pi}{2}}+T_{\frac{\pi}{2}}^{\star}\right), T_{\frac{\pi}{4}}^{\sharp}:=\frac{1}{3}\left((1+\varepsilon) T_{\frac{\pi}{4}}+\widetilde{T}_{\frac{\pi}{2}}\right)$. Multiplying (5) on the left by the diagonal matrix with diagonal $(1, \lambda / \sqrt{2})$ we arrive at a classical but non selfadjoint eigenvalue problem. We analyse its spectrum numerically using the set of 9 trial functions $\Phi_{\beta}(p):=\varphi_{\beta_{1}}\left(p_{1}\right) \varphi_{\beta_{2}}\left(p_{2}\right)$, with $\beta \in\{0.27,1.7,6\}^{2}$ and $\varphi_{\beta_{i}}(u):=\exp \left(-\beta_{i} u^{2}\right), \quad u \in \mathbb{R}$. We get the highest (generalized) eigenvalue $k$ of (5) as a function of $\lambda$ see Figure 1. This shows that

$$
\hat{Z}_{c}(3) \leq 0.86 \text {. }
$$

Although we do believe that this value 0.86 is very likely to be an upper bound on $\hat{Z}_{c}(3)$ we warn the reader that beside the uncertainty due to numerics there is also a gap in our reasoning since we are not yet able to justify our use of variational technics for a non selfadjoint operator. 

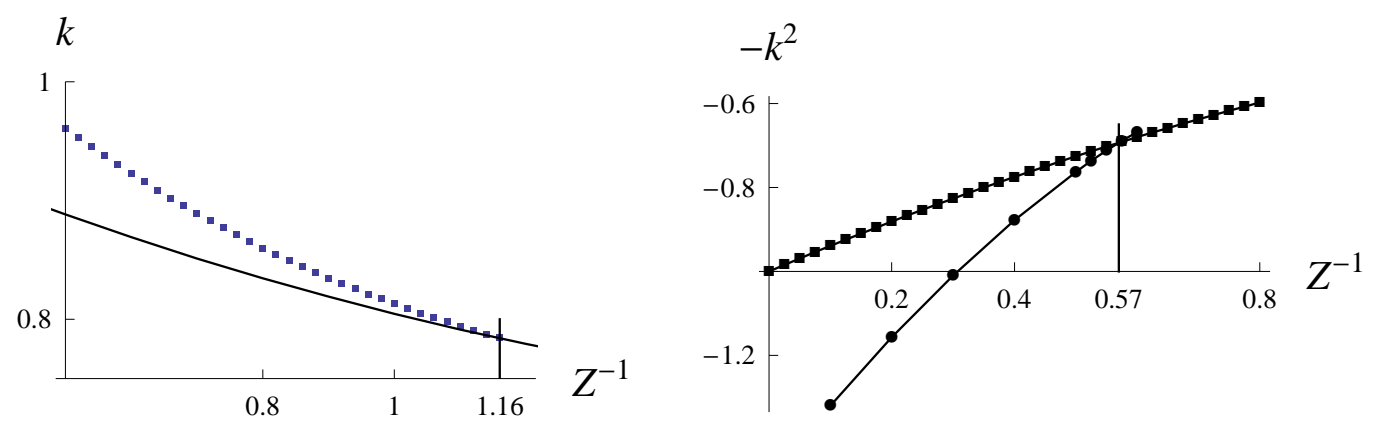

Figure 1. On the letf: the dotted line gives the highest eigenvalue of (5) and the solid line the essential spectrum of (3). On the right: the square dots stand for the energy of the three electrons atoms in $\mathbb{R}^{3}$ with bosonic statistics obtained by the Diffusion Monte Carlo method $[\mathrm{BMBM}]$ and the circle dots for the corresponding two electrons system.

Table 1. Critical ratio

\begin{tabular}{ccc}
\hline$N$ & 2 & 3 \\
\hline$N / Z_{c}(0, N)$ & 2.19 & 1.71 \\
$N / \hat{Z}_{c}(N)$ & 5.31 & $\geq 3.48$ \\
\hline
\end{tabular}

\section{Conclusions}

As announced in the introduction, we display in Table 1 the numbers of electron per unit of nucleus charge at the critical values of these charges.

We have used $Z_{c}(0,2) \simeq 0.9112$ from [StSt, (2.12) and references therein] and $\hat{Z}_{c}(2) \simeq 0.377$ from $[\mathrm{R}] . \hat{Z}_{c}(3)$ has been studied in $\S 3$. In order to estimate the critical charge $Z_{c}(0,3)$ for binding three bosonic electrons we used the Diffusion Monte Carlo method [BMBM], which is known to give exact results, within the statistical uncertainty of the method, for bosonic systems. This method employs a guided random walk that sample the exact, unknown ground state function. To guide the random walk and reduce the statistical uncertainty of the results we used a properly symmetrized guiding function of the kind

$$
\Psi=P_{\text {bose }} \prod_{i=1}^{3} \exp \left(-\left|a_{i}\right| r_{i}\right) \prod_{i<j} \exp \left(b_{i, j} r_{i, j} /\left(1+c_{i, j} r_{i, j}\right)\right) .
$$

The parameters have been optimized for each value of $\lambda=1 / Z$. We performed simulations for $\lambda=0.1,0.2,0.3,0.4,0.5,0.6$. Comparing the energies with the corresponding ones of the 2-body system we located the critical $\lambda$ between 0.5 and 0.6. In order to locate it more precisely we performed additional simulations in that interval, at steps of 0.025 , fitted the results, for both two and three body systems, with quartic polynomials and computed the intersection. We estimate $\lambda_{c}=0.570$, see Figure 1 on the right. 


\section{References}

BMBM. D. Bressanini, G. Morosi, L. Bertini, M. Mella: Stability of Few-Body Systems and Quantum Monte-Carlo Methods Few Body Systems 31, 199 (2002)

BSY. B. Baumgartner, J.-Ph. Solovej, J. Yngvason: Atoms in strong magnetic fields: The high field limit at fixed nuclear charge, Commun. Math. Physics 212 (3), 703 $724(2000)$

BD. R. Brummelhuis, P. Duclos: Effective Hamiltonians for atoms in very strong magnetic fields. J. Math. Phys. 47, 032103 (2006)

CDR1. H. Cornean, P. Duclos, B. Ricaud: Three quantum charged particles interacting through delta potentials, Few-Body Systems 38(2-4), 125-131

CDR2. H. Cornean, P. Duclos, B. Ricaud: On the skeleton method and an application to a quantum scissor Proceedings and Symposia in Pure Mathematics of the AMS 77 (2008) 657-672,

L. Lieb E.H. : Bound on the maximum negative ionization of atoms and molecules. Phys. Rev. A 29, 3018-3028 (1984)

LSY. Lieb H.L., Solovej J. Ph., Yngvason J.: Asymptotics of Heavy Atoms in High Magnetic Fields: I. Lowest landau band Regions. Commun. Pure Appl. Math. 47(4), 513591 (1994)

R. C.M. Rosenthal: Solution of Delta Function Model for Heliumlike Ions Journ. Chem. Phys. 35(5) 2474-2483 (1971)

StSt. F. H. Stillinger and D. K. Stillinger, Nonlinear variational study of perturbation theory for atoms and ions, Phys. Rev. A 10 (1974), 1109-1122 